\title{
Dynamical dimer method for the determination of transition states with ab initio molecular dynamics
}

\author{
Alexander Poddey $*$ and Peter E. Blöch \\ Clausthal University of Technology, Institute of Theoretical Physics \\ Leibnizstrasse 10, D-38678 Clausthal-Zellerfeld, Germany
}

(Dated: October 25, 2018)

\begin{abstract}
A dynamical formulation of the dimer method for the determination of transition states is presented. The method is suited for ab-initio molecular dynamics using the fictitious Lagrangian formulation. The method has been applied to the con-rotatory ring opening of chloro-cyclo-butadiene, an example, where the application of the drag method is problematic.
\end{abstract}

\section{INTRODUCTION}

The concept of the transition state has a fundamental role for the prediction of rate constants of materials processes. The transition state is the lowest point on the energy barrier separating two metastable states representing the initial and final state of a process. The energy difference between the initial state and the transition state is the activation energy. The curvatures of the potential energy surface at the initial state and the transition state provides an estimate of entropic effects [1, 2]. A molecular dynamics simulation starting from the transition state allows one to construct the dynamical trajectory of a reaction in the low-temperature limit. The extension of this latter approach to finite temperatures is straightforward [3].

The determination of transition states is however substantially more difficult than the determination of minima of the total energy surface. A large number of methods have been invented and refined over the last decades (see e.g. the Refs. 4, 5, 6, 7, 8, 9, 10, 11, 12, 13, 14, 15 and the review articles [16, 17]).

Many of these methods require the determination of the second derivatives of the total energy surface. While forces, that is first derivatives of the potential energy surface, are readily accessible from density functional calculations, second derivatives are not, at least not without substantial effort.

An overview of methods which allow to determine transition states solely from force information can be found in [16]. The most simple approach is the drag method, where a one-dimensional constraint forces the system across a reaction barrier. Widely used are also "chain of states" algorithms such as the nudged elastic band (NEB) [10, 11, 12] or the string method [14, 15].

Intermediate between these extremes is the dimer method. [18]. It couples two instances of the system, which we will call monomers. The monomers have a specified distance in configuration space. If the system follows the forces, after inverting of the force parallel to

\footnotetext{
*Electronic address: alexander.poddey@tu-clausthal.de
}

${ }^{\dagger}$ Electronic address: peter.bloechl@tu-clausthal.de the dimer, it evolves to a saddle point of first order, that is the transition state.

The original dimer method [18] applied a two step algorithm. In a first step, the potential energy of the dimer-construct is minimized, excluding parallel motion, following a conjugate gradient approach. Then a large step is taken in the direction parallel to the dimer axis. Recently, an improvement has been suggested which minimizes the number of gradient evaluations [19].

In the context of fictitious-Lagrangian approach to first-principles molecular dynamics (FPMD) [20], such a two-step procedure is not feasible, because the electronic wave functions must be able to follow the atomic motion adiabatically. The fictitious Lagrangian approach requires a larger number of time steps. However the computational cost per time step are minimal. While a first-principles string approach has been published recently [21], the dimer method has not been examined in the framework of ab initio molecular dynamics.

In the present study, we introduce a dynamical formulation of the dimer method. Starting from an extended Lagrangian for the dimer in the configuration space with doubled dimensionality, we derive equations of motion, that, in the presence of dissipation, converge at transition states of first order. An analysis of the trajectories of the dimer close to the stable points provides information on the stability and gives guidance for improving the performance of the method. The method has been implemented in the CP-PAW code and has been applied to the con-rotatory ring-opening of chloro-cyclo-butene, an example where the conventional drag method fails unless special precautions are taken.

The paper is organized as follows: In section II we define the extended Lagrangian for the dimer motion and derive the equations of motion. In section III we analyze stability and the dynamics in the proximity of the stable points. Section IV is devoted to the discretization the equations of motion and the aspects of the actual implementation. In the final section $\mathrm{V}$, the method is applied to the con-rotatory ring opening of cyclo-butene as practical example and compared to the drag method. 


\section{LAGRANGIAN AND EQUATIONS OF MOTION}

In this section we define the Lagrangian underlying the present work and derive the equations of motion. We begin by defining our notation and we introduce a convenient set of coordinates:

The dimer consists of two points in configurational space separated by a fixed distance. We use massweighted coordinates $\mathbf{x}_{i}$ defined as

$$
\mathbf{x}_{i}:=\underline{\mathbf{m}}^{\frac{1}{2}} \mathbf{R}_{i}
$$

where the vectors $\mathbf{R}_{i}$ with $i=1,2$ are the positions of the two monomers forming the dimer in configurational space. For a system with $N$ atoms each vector has $3 N$ dimensions. In addition, each monomer has its own set of electronic wave functions. The mass matrix $\underline{\mathbf{m}}$ is diagonal and the masses of the respective atoms are located on the main diagonal.

It is convenient to introduce a variable transformation into center-of-gravity coordinates $\mathbf{Y}_{1}$ and relative coordinates $\mathbf{Y}_{2}$.

$$
\begin{aligned}
& \mathbf{Y}_{1}=\frac{\mathbf{x}_{1}+\mathbf{x}_{2}}{2} \\
& \mathbf{Y}_{2}=\mathbf{x}_{1}-\mathbf{x}_{2}
\end{aligned}
$$

Loosely speaking $\mathbf{Y}_{1}$ describes the mean structure of the molecule, while $\mathbf{Y}_{2}$ describes the orientation of the dimer.

A dimer has three basic types of motion: motion parallel to the dimer axis $\left(\mathbf{v}_{\|}\right)$, motion perpendicular to the axis $\left(\mathbf{v}_{\perp}\right)$ and rotation about the center of gravity $\left(\mathbf{v}_{\circ}\right)$. In the following, we divide the velocities into the contributions from these basic types of motion. The corresponding velocities are

$$
\begin{aligned}
\mathbf{v}_{\circ} & =\dot{\mathbf{Y}}_{2}=\dot{\mathbf{x}}_{1}-\dot{\mathbf{x}}_{2} \\
\mathbf{v}_{\|} & =\frac{\mathbf{Y}_{2} \otimes \mathbf{Y}_{2}}{d^{2}} \dot{\mathbf{Y}}_{1} \\
& =\left(\mathbf{x}_{1}-\mathbf{x}_{2}\right) \frac{\left(\mathbf{x}_{1}-\mathbf{x}_{2}\right)\left(\dot{\mathbf{x}}_{1}+\dot{\mathbf{x}}_{2}\right)}{2 d^{2}} \\
\mathbf{v}_{\perp} & =\left(1-\frac{\mathbf{Y}_{2} \otimes \mathbf{Y}_{2}}{d^{2}}\right) \dot{\mathbf{Y}}_{1}=\frac{\dot{\mathbf{x}}_{1}+\dot{\mathbf{x}}_{2}}{2}-\mathbf{v}_{\|}
\end{aligned}
$$

The dot denotes the time derivative, and $d$ is the so called 'dimer distance' $d=\sqrt{\mathbf{Y}_{2}^{2}}=\sqrt{\left(\mathbf{x}_{1}-\mathbf{x}_{2}\right)^{2}}$. The symbol $\otimes$ refers to the outer product defined by $(\mathbf{a} \otimes \mathbf{b}) \mathbf{c}=$ $\mathbf{a}(\mathbf{b} \cdot \mathbf{c})$. Note, that $\frac{\mathbf{Y}_{2} \otimes \mathbf{Y}_{2}}{d^{2}}$ is an operator that projects a vector onto the dimer axis.

Now we define our theory by setting up a Lagrangian function in the relative and center-of-gravity coordinates

$$
\begin{aligned}
\mathcal{L}\left(\mathbf{Y}_{1}, \mathbf{Y}_{2}, \dot{\mathbf{Y}}_{1}, \dot{\mathbf{Y}}_{2}\right)= & M_{\circ} \frac{1}{4} \mathbf{v}_{\circ}^{2}+M_{\perp} \mathbf{v}_{\perp}^{2}+M_{\|} \mathbf{v}_{\|}^{2} \\
& -V\left(\underline{\mathbf{m}}^{-\frac{1}{2}}\left[\mathbf{Y}_{1}+\frac{1}{2} \mathbf{Y}_{2}\right]\right) \\
& -V\left(\underline{\mathbf{m}}^{-\frac{1}{2}}\left[\mathbf{Y}_{1}-\frac{1}{2} \mathbf{Y}_{2}\right]\right) \\
& -\bar{\lambda}\left[\mathbf{Y}_{2}^{2}-d^{2}\right]
\end{aligned}
$$

The potential energy is described by the potential energy surface $V(\mathbf{R})$. The velocities $\mathbf{v}_{\circ}, \mathbf{v}_{\perp}$ and $\mathbf{v}_{\|}$are to be treated as functions of $\mathbf{Y}_{1}$ and $\mathbf{Y}_{2}$ and their time derivatives $\dot{\mathbf{Y}}_{1}$ and $\dot{\mathbf{Y}}_{2}$ as defined in Eqs. 41516.

We introduced three scale factors $M_{\circ}, M_{\|}$and $M_{\perp}$, that allow us to scale the masses for the three types of motions independently, that will later be used to accelerate convergence. More importantly, by choosing a negative value of $M_{\|}$, the motion along the dimer direction is inverted, so that the dimer climbs up the barrier.

If the scale factors are equal to one, the conventional kinetic energy is recovered, that is

$$
\frac{1}{4} \mathbf{v}_{\circ}^{2}+\mathbf{v}_{\|}^{2}+\mathbf{v}_{\perp}^{2}=\frac{1}{2} \dot{\mathbf{R}}_{1} \mathbf{m} \dot{\mathbf{R}}_{1}+\frac{1}{2} \dot{\mathbf{R}}_{2} \mathbf{m} \dot{\mathbf{R}}_{2}
$$

Using the method of Lagrange multipliers, a term has been introduced to describe the dimer-distance constraint

$$
\mathbf{Y}_{2}^{2}=d^{2}
$$

which ensures that the dimer distance in mass-weighted coordinates is equal to $d$. The corresponding Lagrange multiplier is denoted by $\bar{\lambda}$.

In addition to the Lagrangian we also define a Rayleigh's dissipation function $\mathcal{D}$

$$
\mathcal{D}=\gamma_{\circ} M_{\circ} \frac{1}{4} \mathbf{v}_{\circ}^{2}+\gamma_{\|} M_{\|} \mathbf{v}_{\|}^{2}+\gamma_{\perp} M_{\perp} \mathbf{v}_{\perp}^{2} .
$$

which allows us to introduce dissipation in a consistent manner.

The Euler-Lagrange equation for the Lagrangian of Eq. (7)and the Rayleigh's Dissipation function from Eq.10 are obtained from

$$
\frac{d}{d t} \frac{\partial \mathcal{L}}{\partial \dot{Y}_{i, n}}-\frac{\partial \mathcal{L}}{\partial Y_{i, n}}+\frac{\partial \mathcal{D}}{\partial \dot{Y}_{i, n}}=0,
$$

where the index $i \in\{1,2\}$ labels the two monomers, and $n \in\{1,3 N\}$ labels the coordinate in configuration space.

The resulting equations of motion have the form

$$
\begin{gathered}
\left(1-\frac{\mathbf{Y}_{2} \otimes \mathbf{Y}_{2}}{d^{2}}\right) M_{\perp}\left(\ddot{\mathbf{Y}}_{1}+\gamma_{\perp} \dot{\mathbf{Y}}_{1}\right) \\
+\left(\frac{\mathbf{Y}_{2} \otimes \mathbf{Y}_{2}}{d^{2}}\right) M_{\|}\left(\ddot{\mathbf{Y}}_{1}+\gamma_{\|} \dot{\mathbf{Y}}_{1}\right) \\
-\frac{1}{2} \mathbf{m}^{-\frac{1}{2}}\left(\mathbf{F}_{1}+\mathbf{F}_{2}\right) \\
+\left(M_{\|}-M_{\perp}\right)\left[\frac{d}{d t}\left(\frac{\mathbf{Y}_{2} \otimes \mathbf{Y}_{2}}{d^{2}}\right)\right] \dot{\mathbf{Y}}_{1}=0
\end{gathered}
$$

and

$$
\begin{aligned}
& M_{\circ} \ddot{\mathbf{Y}}_{2}-\underline{\mathbf{m}}^{-\frac{1}{2}}\left(\mathbf{F}_{1}-\mathbf{F}_{2}\right)+4 \lambda \mathbf{Y}_{2} \\
& +4\left(M_{\perp}-M_{\|}\right) \frac{\mathbf{Y}_{2} \dot{\mathbf{Y}}_{1}}{d^{2}} \dot{\mathbf{Y}}_{1}+\gamma_{\circ} M_{\circ} \dot{\mathbf{Y}}_{2}=0
\end{aligned}
$$


Here we have used the forces acting on the monomers defined as

$$
\mathbf{F}_{i}:=-\left.\nabla\right|_{\mathbf{R}_{i}} V
$$

In equation (13) we absorbed all terms which lead to a force parallel to the dimer axis into the constraint force by redefining the Lagrange multiplier. Thus the variable $\lambda$ used in Eq. 13 differs from the Lagrange multiplier $\bar{\lambda}$ in Eq. 7.

Furthermore, because the dimer system moves on the hyperplane with constant dimer distance, we simplified the final equations by using

$$
\mathbf{Y}_{2}^{2}=d^{2}
$$

and

$$
\frac{\partial}{\partial t} \mathbf{Y}_{2}^{2}=0
$$

Equation (12) describes the motion of the center of gravity of the dimer, which is related to the structure of the molecule. Equation (13) describes the orientational motion of the dimer.

\section{LOCAL STABILITY ANALYSIS}

For $M_{\circ}=M_{\|}=M_{\perp}=1$, the Lagrange function (7) describes a physical system of two masspoints moving in a potential $V$ under the influence of the constraint force that keeps the dimer distance invariant. With positive friction factors $\gamma_{0}, \gamma_{\|}$and $\gamma_{\perp}$ the dimer will come to rest with the center-of-gravity coordinate next to a local minimum. The dimer axis will be aligned nearly parallel to the lowest vibrational eigenmode. The above statements are exactly fulfilled in the limit of vanishing dimer distance.

If we choose a negative value of $M_{\|}$, the motion will become unstable near local minima. Instead, the dimer will be attracted by transition states of first order. A transition state of first order is characterized by the presence of exactly one eigenmode with an imaginary frequency.

These properties of the dynamics have been derived from the following local stability analysis. First we determine the stationary points for the dimer dynamics. Then we investigate the dynamics in the neighborhood of those stationary points.

For this analysis we can replace the potential energy surface by its truncated Taylor expansion at a given point $\mathbf{R}^{(0)}=\mathbf{m}^{-\frac{1}{2}} \mathbf{X}^{(0)}$, namely

$$
\begin{aligned}
V(\mathbf{R})= & V^{(0)}-\mathbf{F}^{(0)} \mathbf{R} \\
& +\frac{1}{2}\left(\mathbf{R}-\mathbf{R}^{(0)}\right) \mathbf{m}^{\frac{1}{2}} \mathbf{D} \mathbf{m}^{\frac{1}{2}}\left(\mathbf{R}-\mathbf{R}^{(0)}\right)
\end{aligned}
$$

with the dynamical matrix $\mathbf{D}$ defined as

$$
D_{i, j}=\left.\frac{1}{\sqrt{m_{i, i}}} \frac{\partial^{2} V}{\partial R_{i} \partial R_{j}}\right|_{\mathbf{R}^{(0)}} \frac{1}{\sqrt{m_{j, j}}}
$$

From Eq. 17 and Eq. 1, we obtain the forces

$$
\mathbf{F}(\mathbf{x})=\mathbf{F}^{(0)}-\mathbf{m}^{\frac{1}{2}} \mathbf{D}\left(\mathbf{x}-\mathbf{x}^{(0)}\right)
$$

\section{A. Stationary points}

If we insert the condition for a stationary point $\dot{\mathbf{Y}}_{i}=$ $\ddot{\mathbf{Y}}_{i}=0$ in the equation of motion Eq. 12, we find that the forces for the two monomers at the stationary point are antiparallel and of the same magnitude. Using the Taylor expansion Eq. [19] in the equation of motion Eq. 12, we find that the center-of-gravity of the dimer $\mathbf{Y}_{1}$ lies at a stationary point of the potential, if the dimer is stationary, that is

$$
\mathbf{F}\left(\mathbf{Y}_{1}\right)=0
$$

Similarly we obtain from Eq. 13 and Eq. 19

$$
\mathbf{D Y}_{2}=-4 \lambda \mathbf{Y}_{2}
$$

Thus the dimer axis points along an eigenvector of the dynamical matrix.

In conclusion we find that the dimer dynamics is stationary, when its center of gravity lies at an extremum or a saddle point of the potential energy surface and when, in addition, the dimer axis points along one of the vibrational eigenmodes.

\section{B. Linearized equation of motion}

Without loss of generality, we choose in the following a coordinate system for which the stationary point of the potential energy surface lies at the origin. Secondly, we denote the eigenvectors of the dynamical matrix as $\mathbf{e}_{i}$, with $\mathbf{e}_{i} \mathbf{e}_{j}=\delta_{i, j}$, and the corresponding eigenvalues with $\omega_{i}^{2}$. The eigenvector, which is parallel to the dimer axis is denoted by $\overline{\mathbf{e}}$ and the corresponding eigenvalue is denoted by $\bar{\omega}^{2}$. Thus, with Eq. 21, we can identify the Lagrange multiplier as $\lambda=-\frac{1}{4} \bar{\omega}^{2}$.

Linearization of the equations of motion Eqs. 12 and 13 about $\mathbf{Y}_{1}=\mathbf{0}$ and $\mathbf{Y}_{2}=\overline{\mathbf{e}} d$ with $\mathbf{F}^{(0)}=0$ yields the following equations for the deviation $\delta \mathbf{Y}_{1}(t)$ and $\delta \mathbf{Y}_{2}(t)$ from the stationary point

$$
\begin{gathered}
(1-\overline{\mathbf{e}} \otimes \overline{\mathbf{e}}) M_{\perp}\left(\delta \ddot{\mathbf{Y}}_{1}+\gamma_{\perp} \delta \dot{\mathbf{Y}}_{1}\right) \\
+(\overline{\mathbf{e}} \otimes \overline{\mathbf{e}}) M_{\|}\left(\delta \ddot{\mathbf{Y}}_{1}+\gamma_{\|} \delta \dot{\mathbf{Y}}_{1}\right)+\mathbf{D} \delta \mathbf{Y}_{1}=0 \\
M_{\circ} \delta \ddot{\mathbf{Y}}_{2}+\mathbf{D} \delta \mathbf{Y}_{2}+\bar{\omega}^{2} \delta \mathbf{Y}_{2}+M_{\circ} \gamma_{\circ} \delta \dot{Y}_{2}=0
\end{gathered}
$$

We project the second equation ,Eq.23, onto the eigenvectors $\mathbf{e}_{i}$ and obtain:

$$
M_{\circ}\left(\mathbf{e}_{i} \delta \ddot{\mathbf{Y}}_{2}\right)+\left(\omega_{i}^{2}-\bar{\omega}^{2}\right)\left(\mathbf{e}_{i} \delta \mathbf{Y}_{2}\right)+M_{\circ} \gamma_{\circ}\left(\mathbf{e}_{i} \delta \dot{Y}_{2}\right)=0(24)
$$

Note, that the motion along $\overline{\mathbf{e}}$ is simple due to the distance constraint. The frequency of the variable $\mathbf{e}_{i} \mathbf{Y}_{2}$ is 
therefore

$$
\omega_{\circ}= \pm \sqrt{\frac{\omega_{i}^{2}-\bar{\omega}^{2}}{M_{\circ}}}
$$

We see that, for a positive mass $M_{\circ}$, the dynamics is stable only, if $\bar{\omega}^{2}$ is the lowest eigenvalue of the dynamical matrix. Thus the dimer axis will always orient along the eigenvector with the lowest eigenvalue.

Now, we project Eq. 22 onto the eigenvector $\overline{\mathbf{e}}$ of the dynamical matrix

$$
M_{\|}\left(\overline{\mathbf{e}} \delta \ddot{\mathbf{Y}}_{1}\right)+\bar{\omega}^{2}\left(\overline{\mathbf{e}} \delta \mathbf{Y}_{1}\right)+M_{\|} \gamma_{\|}\left(\overline{\mathbf{e}} \delta \dot{\mathbf{Y}}_{1}\right)=0
$$

which yields the translational motion of the dimer along the dimer axis. This equation will be responsible for the ascent to a saddle point. The eigenfrequency of the variable $\overline{\mathbf{e}} \mathbf{Y}_{1}$ is

$$
\omega_{\|}= \pm \frac{\bar{\omega}}{\sqrt{M_{\|}}}
$$

For a negative mass $M_{\|}$, which is the choice for a transition state search, Eq. 26 is stable only if the dimer is oriented along an unstable vibrational mode of the potential energy surface, that is with the dimer oriented along an eigenvector $\overline{\mathbf{e}}$ with an imaginary frequency.

Now, we project Eq. 22 onto the eigenvectors $\mathbf{e}_{i}$ with $\mathbf{e}_{i} \perp \overline{\mathbf{e}}$ and we obtain

$$
M_{\perp}\left(\mathbf{e}_{i} \delta \ddot{\mathbf{Y}}_{1}\right)+\omega_{i}^{2}\left(\mathbf{e}_{i} \delta \mathbf{Y}_{1}\right)+M_{\perp} \gamma_{\perp}\left(\mathbf{e}_{i} \delta \dot{\mathbf{Y}}_{1}\right)=0
$$

which represents the translational motion perpendicular to the dimer axis. The translational motion is related to an optimization of the atomic structure. The frequency of the variable $\mathbf{e}_{i} \mathbf{Y}_{1}$ with $\mathbf{e}_{i} \perp \overline{\mathbf{e}}$ is

$$
\omega_{\perp}=\frac{\omega_{i}}{\sqrt{M_{\perp}}}
$$

From Eqs. 25, 27 and 29 we find that the dimer with positive $M_{\circ}$ and $M_{\perp}$ and negative $M_{\|}$will only come to rest at transition states of first order, that is, if $\bar{\omega}^{2}<0$ and if all other eigenvalues are positive. In that case the dimer axis points along the eigenvector corresponding to the imaginary frequency, that is, across the saddle point.

\section{Masses and Frictions}

We will later solve the equations of motion 12, 13 with the Verlet algorithm. The Verlet algorithm for a harmonic oscillator with a frequency $\omega$ becomes unstable, if the time step $\Delta$ used for the discretization of the equation of motion is smaller than $2 / \omega$. The frequency of the discretized motion is accurate to within $1 \%$ if $\omega \Delta<\frac{\pi}{5}$. Thus, it is important to understand the vibrational spectrum, shown schematically in Fig. 1. of the motion in the potential.

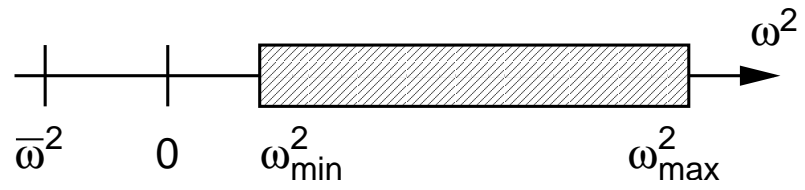

FIG. 1: Sketch of the eigenvalue spectrum of the dynamical matrix at the saddle point of first order with the definition of $\omega_{\min }$ and $\omega_{\max }$.

Thus, the stability limit requires us to choose the masses as

$$
\begin{aligned}
& M_{\|}<\frac{\bar{\omega}^{2} \Delta^{2}}{8} \\
& M_{\perp}>\frac{\omega_{\max }^{2} \Delta^{2}}{8} \\
& M_{\circ}>\frac{\left(\omega_{\max }^{2}-\omega_{\min }^{2}\right) \Delta^{2}}{4}
\end{aligned}
$$

In order to minimize the number of time steps, it is desirable to choose the masses close to these limits.

Let us now obtain an a priori estimate of these limits: (1) For practical purposes, we can make the assumption that $\omega_{\min }<<\omega_{\max }$ and thus we set $\omega_{\min }$ to zero. (2) The highest vibrational mode is that of $\mathrm{H}_{2}$, with a frequency of $4400 \mathrm{~cm}^{-1}$. Frequencies for bond-stretch vibrations are of the order $1000 \mathrm{~cm}^{-1}$. These numbers are reasonable upper estimates for $\omega_{\max }$. (3) The absolute value of the imaginary frequency at the saddle point is typically in the range of the high-lying real-frequency modes, that is comparable to $\omega_{\max }$. Hence $\bar{\omega}^{2} \approx-\omega_{\max }^{2}$. With these assumptions we obtain the following, recommended values for the masses

$$
\begin{aligned}
M_{\|} & =-\kappa \Delta^{2} \\
M_{\perp} & =\kappa \Delta^{2} \\
M_{\circ} & =2 \kappa \Delta^{2}
\end{aligned}
$$

where $\kappa=\left(6 \cdot 10^{-3} \text { a.u. }\right)^{2}$ is a recommended constant based on the $\mathrm{H}_{2}$ vibration.

Let us now turn our attention to the friction values. The goal is to reach an optimum convergence at the stationary point of the dimer. For a damped harmonic oscillator

$$
m \ddot{x}=-m \omega_{0}^{2} x-m \gamma x
$$

the fastest decay rate as function of the friction is obtained for critical damping, that is in between the oscillatory and the over-damped regime. Critical damping is 
obtained for a friction of $\gamma=2 \omega_{0}$. Thus we choose

$$
\begin{aligned}
\gamma_{\|} & =2 \sqrt{\frac{\bar{\omega}^{2}}{M_{\|}}} \\
2 \sqrt{\frac{\omega_{\text {min }}^{2}}{M_{\perp}}} & <\gamma_{\perp}<2 \sqrt{\frac{\omega_{\text {max }}^{2}}{M_{\perp}}} \\
2 \sqrt{\frac{\omega_{\text {min }}^{2}-\bar{\omega}^{2}}{M_{\circ}}}<\gamma_{\circ} & <2 \sqrt{\frac{\omega_{\text {max }}^{2}-\bar{\omega}^{2}}{M_{\circ}}}
\end{aligned}
$$

Note, that a too high friction freezes out those modes in the over-damped regime. Thus it is usually better to use a friction near the lower bound of the sensible regime.

\section{NUMERICAL INTEGRATION OF THE EQUATIONS OF MOTION}

\section{A. Discretization of the equations of motion}

The equations of motion Eqs. 1213 are nonlinear in the velocities. This leads to a nonlinear equation for the discretized equation of motion. We tackle the problem by iterating on the nonlinear terms in the velocities.
We set up the discretized equations of motion, while treating the terms nonlinear in the velocities as an abstract force.

$$
\begin{aligned}
\mathbf{G}_{1} & :=-\left(M_{\|}-M_{\perp}\right)\left[\frac{d}{d t}\left(\frac{\mathbf{Y}_{2} \otimes \mathbf{Y}_{2}}{d^{2}}\right)\right] \dot{\mathbf{Y}}_{1} \\
\mathbf{G}_{2} & :=4\left(M_{\|}-M_{\perp}\right) \frac{\mathbf{Y}_{2} \dot{\mathbf{Y}}_{1}}{d^{2}} \dot{\mathbf{Y}}_{1}
\end{aligned}
$$

The equations of motion are discretized according to the Verlet algorithm:

$$
\begin{aligned}
& \dot{y} \rightarrow \frac{y(+)-y(-)}{2 \Delta} \\
& \ddot{y} \rightarrow \frac{y(+)-2 y(0)+y(-)}{\Delta^{2}}
\end{aligned}
$$

where $\Delta$ is the discretization time step, and where we used the short-hand notation

$$
\begin{aligned}
y(+) & =y(t+\Delta) \\
d y(0) & =y(t) \\
y(-) & =y(t-\Delta)
\end{aligned}
$$

We obtain from Eq. 12

$$
\begin{array}{r}
\left(1-\frac{\mathbf{Y}_{2} \otimes \mathbf{Y}_{2}}{d^{2}}\right) \frac{M_{\perp}}{\Delta^{2}}\left\{\left(1+a_{\perp}\right) \mathbf{Y}_{1}(+)-2 \mathbf{Y}_{1}(0)+\left(1-a_{\perp}\right) \mathbf{Y}_{1}(-)\right\} \\
+\left(\frac{\mathbf{Y}_{2} \otimes \mathbf{Y}_{2}}{d^{2}}\right) \frac{M_{\|}}{\Delta^{2}}\left\{\left(1+a_{\|}\right) \mathbf{Y}_{1}(+)-2 \mathbf{Y}_{1}(0)+\left(1-a_{\|}\right) \mathbf{Y}_{1}(-)\right\} \\
-\frac{1}{2} \mathbf{m}^{-\frac{1}{2}}\left(\mathbf{F}_{1}+\mathbf{F}_{2}\right)-\mathbf{G}_{1}=0
\end{array}
$$

where $a_{\perp}=\frac{\gamma_{\perp} \Delta}{2}$ and $a_{\|}=\frac{\gamma_{\|} \Delta}{2}$. In the projectors we The result is have dropped the argument of $\mathbf{Y}_{2}(0)$.

Eq. 47 can be resolved for $\mathbf{Y}_{1}(+)$ by multiplication with

$$
\begin{aligned}
& \left(1-\frac{\mathbf{Y}_{2} \otimes \mathbf{Y}_{2}}{d^{2}}\right) \frac{\Delta^{2}}{M_{\perp}\left(1+a_{\perp}\right)} \\
& +\left(\frac{\mathbf{Y}_{2} \otimes \mathbf{Y}_{2}}{d^{2}}\right) \frac{\Delta^{2}}{M_{\|}\left(1+a_{\|}\right)}
\end{aligned}
$$

$$
\begin{aligned}
\mathbf{Y}_{1}(+)= & \left(1-\frac{\mathbf{Y}_{2} \otimes \mathbf{Y}_{2}}{d^{2}}\right)\left\{\frac{2}{1+a_{\perp}} \mathbf{Y}_{1}(0)-\frac{1-a_{\perp}}{1+a_{\perp}} \mathbf{Y}_{1}(-)+\left(\frac{1}{2} \mathbf{m}^{-\frac{1}{2}}\left(\mathbf{F}_{1}+\mathbf{F}_{2}\right)+\mathbf{G}_{1}\right) \frac{\Delta^{2}}{M_{\perp}\left(1+a_{\perp}\right)}\right\} \\
& +\left(\frac{\mathbf{Y}_{2} \otimes \mathbf{Y}_{2}}{d^{2}}\right)\left\{\frac{2}{1+a_{\|}} \mathbf{Y}_{1}(0)-\frac{1-a_{\|}}{1+a_{\|}} \mathbf{Y}_{1}(-)+\left(\frac{1}{2} \mathbf{m}^{-\frac{1}{2}}\left(\mathbf{F}_{1}+\mathbf{F}_{2}\right)+\mathbf{G}_{1}\right) \frac{\Delta^{2}}{M_{\|}\left(1+a_{\|}\right)}\right\}
\end{aligned}
$$


first propagate without the force of constraint to obtain

$$
\begin{aligned}
\overline{\mathbf{Y}}_{2}= & \frac{2}{1+a_{\circ}} \mathbf{Y}_{2}(0)-\frac{1-a_{\circ}}{1+a_{\circ}} \mathbf{Y}_{2}(-) \\
& +\left(\mathbf{m}^{-\frac{1}{2}}\left(\mathbf{F}_{1}-\mathbf{F}_{2}\right)+\mathbf{G}_{2}\right) \frac{\Delta^{2}}{M_{\circ}\left(1+a_{\circ}\right)}
\end{aligned}
$$

with $a_{\circ}=\frac{\gamma_{\circ} \Delta}{2}$. The new vector $\mathbf{Y}_{2}(+)$ is related to $\bar{Y}_{2}$ by the constraint force and the Lagrange parameter $\lambda$ according to

$$
\mathbf{Y}_{2}(+)=\overline{\mathbf{Y}}_{2}-4 \lambda \mathbf{Y}_{2}(0) \frac{\Delta^{2}}{M_{\circ}\left(1+a_{\circ}\right)}
$$

The Lagrange parameter is then adjusted so that the constraint $Y_{2}^{2}(+)=d^{2}$, namely a given dimer length, is satisfied.

In the first iteration we estimate this forces $\mathbf{G}_{1}$ and $\mathbf{G}_{2}$ from the previous iterations or we set them to zero. Then we propagate the positions, which provides us with a better estimate for the nonlinear terms. This loop is then iterated to convergence.

\section{B. Restricting the orientation of the dimer}

It will be important to limit the degrees of freedom, in which the two monomers may differ. This implies restricting the orientation of the dimer to a space with lower dimensionality. The reason is to avoid that the dimer converges at irrelevant saddle-points, which are not of interest. This is a common problem for complex systems, that exhibit many minima and saddle points. This restriction is accomplished easily, by setting the corresponding components of $\mathbf{Y}_{2}$ in Eq. 50 to zero.

\section{APPLICATION: CONROTATORY RING OPENING OF 1-CHLORO-2-CYCLOBUTENE}

In order to demonstrate the performance of the dimer method described above, we have chosen a prototypical system for tests of transition state searches, namely the ring opening of cyclobutene. The isomerization of cyclobutene to cis-butadiene is the prototypical example of concerted stereospecific reactions. The underlying processes have been studied and discussed extensively by several groups [23, 24, 25, 26] (and references therein). The potential energy surfaces of the con- as well as of the disrotatory isomerization mechanisms exhibit principal structures, which render the determination of the corresponding transition states complicated [24].

In order to avoid special effects due to the high symmetry of cyclobutene, that would be untypical for other molecules, we explored 1-chloro-2-cyclobutene. This molecule and the two relevant reaction products are shown in Figure 2 ,

Computational details of our calculations are given in appendix $\mathrm{A}$. The coordinates of the structures are supplied with the supplementary material.

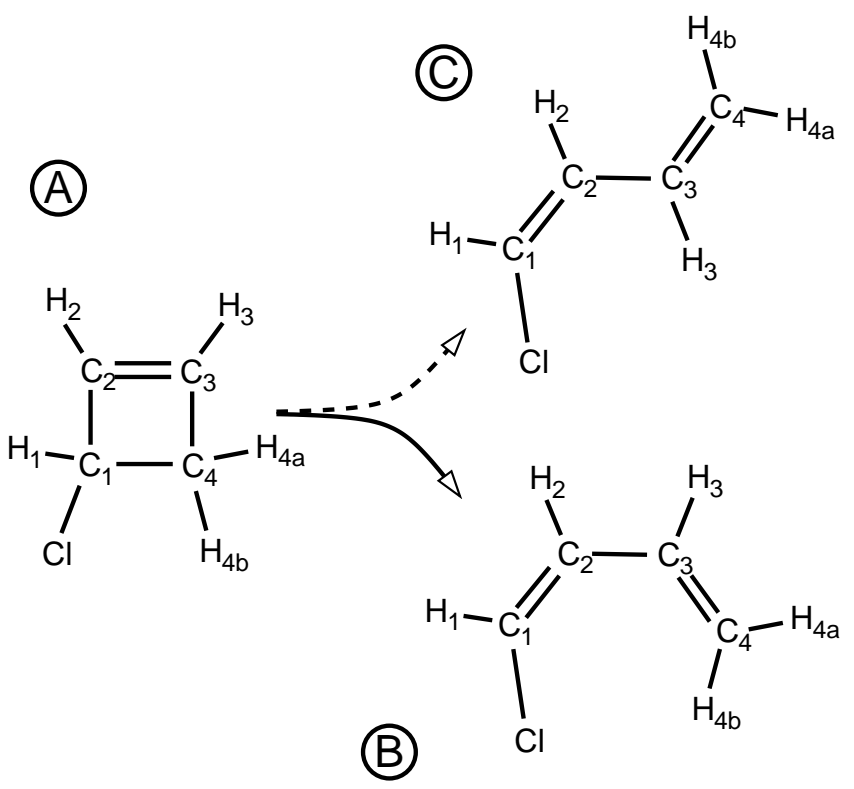

FIG. 2: Initial state, 1-chloro-2-cyclobutene (A), and final state, 1-chloro-buta-1,3-diene (B), for the conrotatory ring opening of chlorocyclobutene. Also shown is the product of the disrotatory ring opening, trans-1-chloro-buta-1,3-diene (C).

\section{A. Drag Calculations}

In this section we investigate the reaction using the drag method. Because of its simplicity, the drag method is widely used for transition state search. However, the drag method fails for a number of systems. In such cases the energy exhibits a hysteresis effect, that is, different energy profiles are obtained when dragging the reaction coordinate in the opposite directions. The ring opening of cyclobutene is one example where the drag method exhibits a hysteresis effect.

In the drag method one specifies a one-dimensional reaction coordinate. Then one maps the energy profile along the reaction coordinate and determines the highest point as the transition state. Each point along the energy profile corresponds to the local energy minimum on a hyperplane perpendicular to the reaction coordinate. In our case we selected the reaction coordinate by the difference between initial and final state. A hyperplane with a specified value $c$ of the reaction coordinate is given by

$$
\frac{\left(\mathbf{R}_{B}-\mathbf{R}_{A}\right)\left(\mathbf{R}-\mathbf{R}_{A}\right)}{\left(\mathbf{R}_{B}-\mathbf{R}_{A}\right)^{2}}=c
$$

Hence the value of the reaction coordinate is zero for the initial state (A) and one for the final state (B).

In addition to the reaction coordinate we imposed six additional constraints to avoid translations and rotations 
of the molecule. We have chosen

$$
\begin{aligned}
\frac{1}{2}\left(\mathbf{R}\left(C_{2}\right)+\mathbf{R}\left(C_{3}\right)\right) & =0 \\
z\left(C_{1}\right)=z\left(C_{4}\right)=\frac{1}{2}\left(x\left(C_{1}\right)+x\left(C_{4}\right)\right) & =0
\end{aligned}
$$

Where the coordinates correspond to the atoms given in parenthesis. The notation follows Figure 2.

When the energy profile is determined by varying the reaction coordinate in small steps, once from zero to one and then from one to zero, we obtain the hysteresis shown in Fig. 3. The highest point of the energy profile is not the transition state. Instead, the highest point of each branch is an upper bound for the activation energy, while the crossing of the two branches is a lower bound.

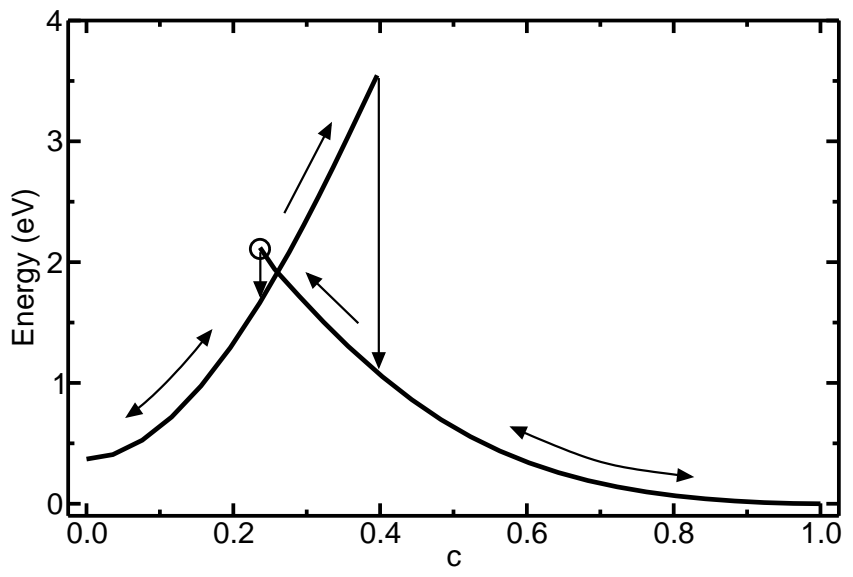

FIG. 3: Potential energy relative to the energy of final state (B) over reaction coordinate from a forward and backward calculation using the drag method. The true transition state (determined using the dynamical dimer method) is denoted by a circle.

In order to show the underlying reason for the hysteresis, we show in Fig. 4 the total energy surface in a two-dimensional hypersurface. The two axes are the reaction coordinate and the coordinate defined by the two isoenergetic structures at the crossing of the two branches of the energy profile in Fig. 3. For each point in the twodimensional plot the energy is at a local minimum of the $(3 N-2)$-dimensional hypersurface.

We see that the drag method in the forward direction leads into a side valley that leads further onto a ridge. The point where this path becomes unstable, and from where it leads down into the valley of the product state, lies past the transition state. In the backward direction a similar instability occurs, even though closer to the reaction coordinate of the transition state.

Note that the two configuration $A^{\dagger}$ and $B^{\dagger}$, that mark the crossing of the two branches in the energy profile shown in Fig. 3, lie far from the transition state. For further information see Table 1 .

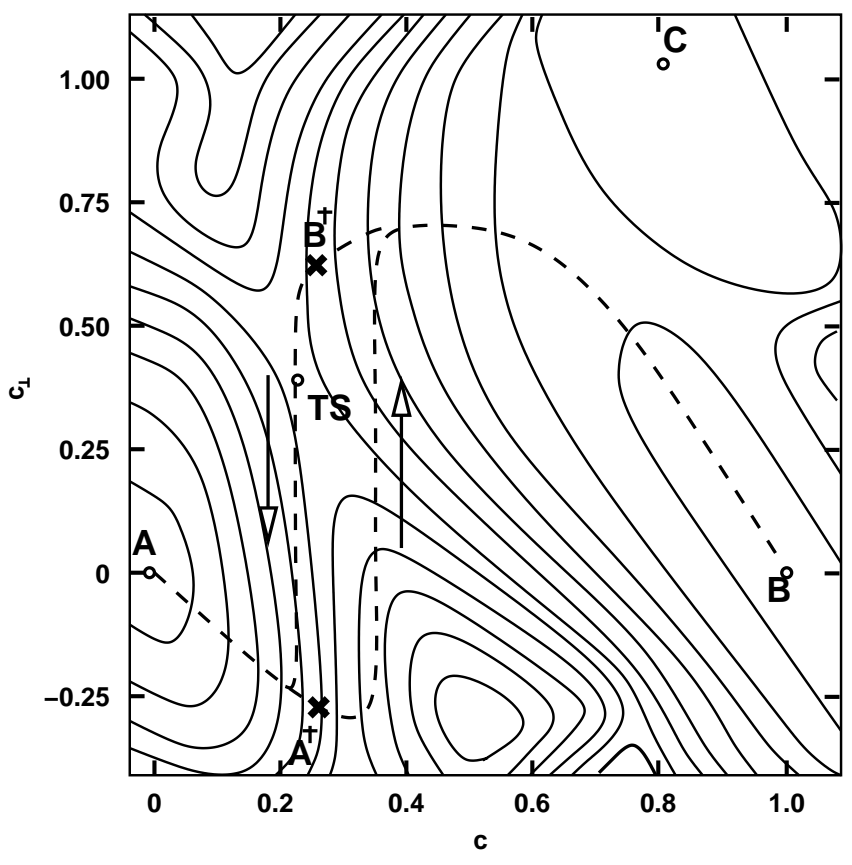

FIG. 4: Sketch of the potential energy surface of the conrotatory isomerization of 1-chloro-2-cyclo-butene to 1-chlorobuta-1,3-diene. The dashed lines trace the paths obtained from drag calculations for the transitions $\mathrm{A} \rightarrow \mathrm{B}$ and $\mathrm{B} \rightarrow \mathrm{A}$, respectively. The crosses denote the structures $A^{\dagger}$ and $B^{\dagger}$. TS corresponds to the transition state.

\section{B. Dynamical dimer calculations}

In this section we describe technical issues for dynamical dimer calculations and demonstrate their performance.

We use the fictitious Lagrangian formulation of abinitio molecular dynamics. This implies that wave function coefficients and nuclei obey Newton's equation of motion, to which we added a friction, which allows to quench the system.

Special attention should be given to the wave function dynamics. The wave-function cloud tied to the atoms results in an increased effective mass of the nuclei. Furthermore, a friction applied to the wave function dynamics acts like an effective friction on the nuclear motion. For a normal ground-state search, this is not problematic. In our case however, the mass for the motion parallel to the dimer direction is inverted. Thus the dimer accelerates opposite to the direction of the force. Thus a friction acting on the dimer via the electrons leads to an velocitydependent acceleration of the dimer, that may cause an instability of the dimer motion.

The detrimental effect of the wave function motion can be avoided by choosing a sufficiently small mass for the wave function dynamics or by artificially increasing the atomic masses. In our study, we used a mass of $50 \mathrm{u}$ for all atoms. The wave functions have been kept close to 
the ground state by applying a friction that dissipates $2 \%$ of the kinetic energy in each time step.

The dimension-less masses for the dimer motion have been chosen to

$$
\begin{aligned}
M_{\|} & =-1.00 \\
M_{\perp} & =+1.00 \\
M_{\circ} & =+0.25 .
\end{aligned}
$$

The small value for $M_{\circ}$ has been chosen to speed up the reorientation of the dimer.

In order to avoid instabilities, we found it useful to introduce an upper limit to the kinetic energy individually for the rotational, perpendicular and parallel motion. These limits were enforced by adjusting the frictions accordingly. The limit for the kinetic energy $E_{k i n, \max }$ is translated into an upper "temperature" $T_{\max }$ according to

$$
\frac{1}{2} g k_{B} T_{\max }=E_{k i n, \max }
$$

where $g$ is the number of degrees of freedom in the corresponding motion type, $k_{B}$ is Boltzmann's constant.

Within the limits of the enforced maximum kinetic energies, we adapted the friction dynamically in each step to come close to the limit of critical damping. The latter results in the best possible convergence behavior.

Furthermore, the friction is increased to a higher value specified by the user, if the dimer moves away from the stable point. The decision to increase the friction has been made on the basis of the actual forces and velocities. In our simulation we have chosen this friction so that the energy dissipated per time step corresponds to $20 \%$ of the kinetic energy of the corresponding type of motion.

When starting the dimer simulation, it is important to optimize the dimer orientation, i.e. $\mathbf{Y}_{1}$, first, before the mean dimer configuration $\mathbf{Y}_{2}$ changes appreciably. This is because the mean dimer configuration only approaches the transition state, if the dimer orientation is sufficiently aligned along the unstable vibrational mode of the transition state.

The dimer orientation can be optimized in different ways. (1) Starting from initial dimer coordinates, the mean dimer configuration, $\mathbf{Y}_{1}$, is constrained to the initial value, while the orientation, $\mathbf{Y}_{2}$, is fully optimized. Only after this optimization also the mean configuration is allowed to move. (2) Starting from one monomer configuration, the second monomer is constructed from the first by letting it follow the forces until the desired dimer length is obtained. We will refer to this technique as the "growing-dimer technique" (3) Starting from a dimer with the monomers being identical to the two metastable minima, the dimer length is successively shortened, while the dimer position is optimized. Results for all three optimization strategies can be found in Table []

We found that the third strategy suffers from the fact that additional effort is required to contract the dimer length to the desired value. It also suffers from an instability caused by strong anharmonic effects.
The growing-dimer technique has the advantage that the second monomer is constructed dynamically from the first, without the need of an independent optimization of the wave functions. In the growing dimer technique the dimer orientation is optimized automatically as soon as the targeted dimer length is reached. In the first strategy the orientation is obtained during the first iterations with a constrained mean dimer configuration.

We will discuss here the first strategy. The initial structure of the dimer is given by a mean dimer configuration midway between the two metastable states (A) and (B). The dimer length is chosen such that the distance of the two monomers in coordinate space is $0.33 \AA$.

During the first 700 time steps, the dimer orientation has been optimized. Here the mean dimer configuration has not been constrained, but the maximum temperature for the perpendicular motion has been kept at a small value of $10 \mathrm{~K}$. For the parallel and rotational motion, the maximum temperature has been set to $500 \mathrm{~K}$. After the first 700 time steps the maximum temperature for the perpendicular motion has been increased to $500 \mathrm{~K}$. In Figs. 516 we show the convergence of the forces for the parallel, rotational and perpendicular motion. Good convergence is reached after 2000 time steps. The estimates for the transition state energy and the deviation of the mean dimer configuration from the transition state is given in Table 【.

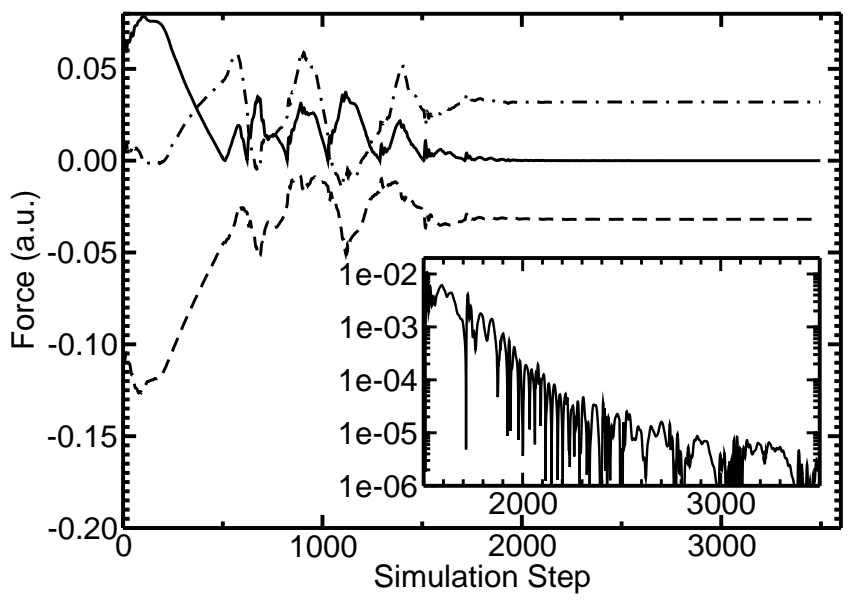

FIG. 5: Forces over time-step for a calculation following optimzation strategy (1) described in the text. The dashed and dash-dotted line show the forces parallel to the dimer axis for image one and two, respectively. The full line corresponds to the resulting force acting on the center of gravity of the dimer. The logarithmic scale of the inset provides detailed information for the latter.

\section{SUMMARY}

A formulation of the dimer method for searching transition states is presented which can be used in ab-initio 


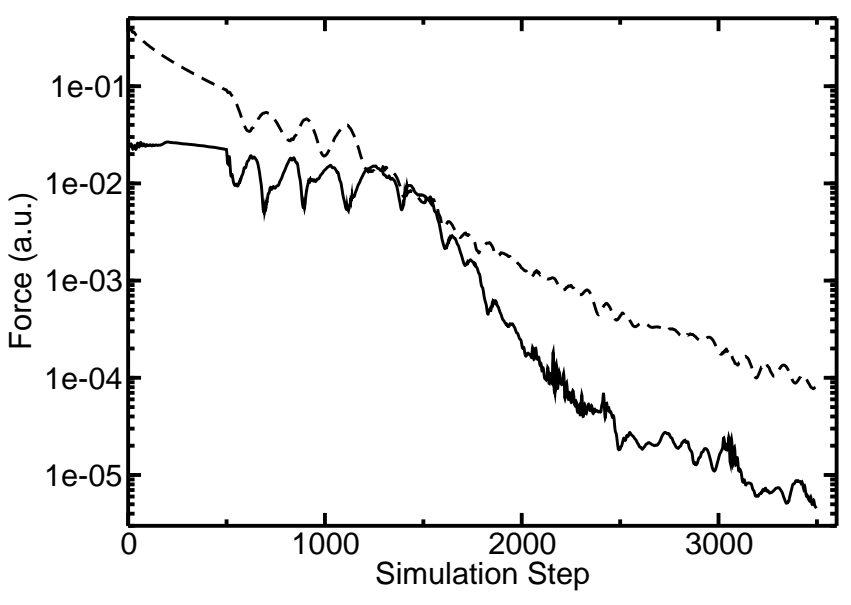

FIG. 6: Rotational (full line) and perpendicular (dashed line) part of the forces acting on the dimer over time-steps for a calculation following optimzation strategy (1) described in the text.

TABLE I: Predicted transition-state energy (relative to final state (B)) and deviation from the exact transition state for the con-rotatory isomerization of 1-chloro-2-cyclo-butene to 1-chloro-buta-1,3-diene. The results include three different dynamical dimer optimization strategies ((1), (2) and (3)) described in the text and the forward as well as the backward drag calculation shown in Fig. 3.

\begin{tabular}{|c|c|c|}
\hline & Predicted Energy $(\mathrm{eV})$ & \begin{tabular}{|l|l}
$\mathbf{R}^{T S}-\mathbf{R}$ & $(\AA)$ \\
\end{tabular} \\
\hline Transition State $^{a}$ & 2.110 & - \\
\hline \multicolumn{3}{|l|}{ (1) } \\
\hline 1000 steps & 2.110 & 1.00 \\
\hline 1500 steps & 2.111 & 0.85 \\
\hline 2000 steps & 2.111 & 0.71 \\
\hline \multicolumn{3}{|l|}{ (2) } \\
\hline 1000 steps & 2.258 & 0.62 \\
\hline 1500 steps & 2.116 & 0.37 \\
\hline 2000 steps & 2.110 & 0.36 \\
\hline \multicolumn{3}{|l|}{$(3)$} \\
\hline 1000 steps & 2.133 & 1.58 \\
\hline 1500 steps & 2.112 & 0.85 \\
\hline 2000 steps & 2.110 & 0.85 \\
\hline $\mathrm{TS} A \rightarrow B$ & 3.461 & 2.57 \\
\hline $\mathrm{TS} B \rightarrow A$ & 2.120 & 0.56 \\
\hline
\end{tabular}

${ }^{a}$ Determined by the use of a well converged dynamical dimer calculation with final dimer distance of $0.125 \AA$.

molecular dynamics simulations using a fictitious Lagrangian. The dimer method is successful in cases where the widely used drag method fails. We investigate the dynamics close to the stable points of the dimer analytically. In addition we demonstrate the performance of our implementation using a practical example typically used as test case for transition state search algorithms, namely the con-rotatory ring opening of (chloro-) cyclo-butene.

\section{APPENDIX A: COMPUTATIONAL DETAILS}

We performed density-functional calculations 27, 28] based on the projector augmented wave (PAW) method 29, 30]. The gradient-corrected PBE[31] functional was used for exchange and correlation. The PAW method is a frozen-core all-electron method. Like other plane-wave based methods, the PAW method leads to the occurrence of artificial periodic images of the structures. This effect was avoided by explicit subtraction of the electrostatic interaction between them. 32] Wave function overlap was avoided by choosing the unit cell large enough to keep a distance of more than $6 \AA$ between atoms belonging to different periodic images. We used a plane wave cutoff of 30 Ry for the auxiliary wave functions of the PAW method. The following sets of projector functions were employed, Cl 2s2p1d, C 2s2p1d, H $2 \mathrm{~s} 1 \mathrm{p}$, which provides the number of projector functions per angular momentum magnetic quantum number $m$ in each main angular momentum channel $\ell$.

Atomic structures were optimized by damped CarParrinello 33 molecular dynamics. We used a time-step of 10 a.u. (2.5 fs) for all except the dynamical dimer calculations. See section $\sqrt{\mathrm{VB}}$ for further details. The convergence was tested by monitoring if the total energy change remains below $10^{-5}$ Hartree during a simulation of 500 time steps. During the simulation for the convergence test, no friction was applied to the atomic motion and the friction on the wave function dynamics was chosen sufficiently low to avoid a noticeable effect on the atomic motion. 
[1] H. Eyring, J. Chem. Phys. 3, 107 (1934).

[2] G. Vineyard, J. Phys. Chem. Solids 3, 121 (1957).

[3] J. Keck, Discuss. Faraday Soc. 33, 1962 (1962).

[4] D. Poppinger, Chem. Phys. Lett. 35, 550 (1975).

[5] C. J. Cerjan and W. H. Miller, J. Chem. Phys. 75, 2800 (1981).

[6] J. Baker, J. Comp. Chem. 7, 385 (1986).

[7] J. Simons, P. Jorgensen, H. Taylor, and J. Ozment, J. Phys. Chem. 87, 2745 (1983).

[8] A. Banerjee, N. Adams, J. Simons, and R. Shepard, J. Phys. Chem. 89, 52 (1985).

[9] L. J. Munro and D. J. Wales, Phys. Rev. B 59, 3969 (1999).

[10] H. Jonsson, G. Mills, and K. W. Jacobsen, Classical and Quantum Dynamics in Condensed Phase Simulations, Ed. B.J. Berne, G.Ciccotti and D.F.Coker, World Scientific p. 385 (1998).

[11] G. Mills and H. Jonsson, Phys. Rev. Lett. 72, 1124 (1994).

[12] G. Mills, H.Jonsson, and G. Schenter, Surf. Sci. 324, 305 (1995).

[13] R. Malek and N. Mousseau, Phys. Rev. E 62, 7723 (2000).

[14] W. E, W. Ren, and E. Vanden-Eijnden, Phys. Rev. B 66, 52301 (2002).

[15] B. Peters, A. Heyden, A. T. Bell, and A. Chakraborty, J. Chem. Phys. 120, 7877 (2004).

[16] G. Henkelman, G. Johannesson, and H. Jonsson, Progress on Theoretical Chemistry and Physics, Ed. S. D. Schwartz, Kluwer Academic Publishers p. 269 (2000).

[17] H. B. Schlegel, J. Comput. Chem. 24, 1514 (2003).
[18] G. Henkelman and H. Jonsson, J. Chem. Phys. 111, 7010 (1999).

[19] R. A. Olsen, G. H. Kroes, G. Henkelman, A. Arnoldsson, and H.Jonsson, J. Chem. Phys. 121, 9776 (2004).

[20] R. Car and M. Parrinello, Phys. Rev. Lett. 55, 2471 (1985).

[21] Y. Kanai, A. Tilocca, A. Selloni, and R. Car, J. Chem. Phys. 121, 3359 (2004).

[22] J.-P. Ryckaert, G. Ciccotti, and H. J. C. Berendsen, J. Comp. Phys. 23, 327 (1977).

[23] R. B. Woodward and R. Hoffmann, J. Am. Chem. Soc. 87, 395 (1965).

[24] M. J. S. Dewar and S. Kirschner, J. Am. Chem. Soc. 93, 4292 (1971).

[25] J. Breulet and H. F. Schaefer, J. Am. Chem. Soc. (1981).

[26] J. M. Olivia, J. Gerratt, P. B. Karadakov, and D. L. Cooper, J. Chem. Phys. 107, 8917 (1997).

[27] P. Hohenberg and W. Kohn, Phys. Rev. 136, B864 (1964).

[28] W. Kohn and L. Sham, Phys. Rev. 140, A1133 (1965).

[29] P. E. Blöchl, Phys. Rev. B 50, 17953 (1994).

[30] P. E. Blöchl, C. Först, and J. Schimpl, Bull. Mater. Sci. 26, 33 (2003).

[31] J. Perdew, K. Burke, and M. Ernzerhof, Phys. Rev. Lett. 77, 3865 (1996).

[32] P. E. Blöchl, J. Chem. Phys. 103, 7422 (1986).

[33] R. Car and M. Parrinello, Phys. Rev. Lett. 55, 2471 (1985). 